\title{
Design and Performance Analysis of low power and high throughput of analog data compression and decompression using ANN in 32nm FinFET Technology
}

\author{
${ }^{1}$ G. K. Venkatesh, ${ }^{2}$ S.Bhargavi, ${ }^{3}$ Basavaraj V. Hiremath, ${ }^{4}$ Anil Kumar C. \\ ${ }^{1}$ Dept of ECE, CBIT, India \\ ${ }^{2}$ Dept of ECE, S J C Institute of Technology, Chickballapur, India \\ ${ }^{3}$ Department of Medical Electronics Engineering, M S Ramaiah Institute of Technology, Bengaluru, \\ India \\ ${ }^{4}$ Dept of ECE, R.L.J.I.T Doddaballapur, India
}

Received: February 4, 2021. Revised: July 3, 2021. Accepted: July 23, 2021. Published: July 28, 2021.

\begin{abstract}
The development and fabrication of integrated circuits for the applicational areas of VLSI such as processing of the signal, medicine tomography, telecommunication turn out to be a novel technology for the upcoming innovations. The fabrication of IC's is attributable to the methodology in the technology of VLSI and when compared to artificial Neural Network, the genetic performance of these productions is approximately the same and are typically employed for diagnosing the syndrome, compression as well as the decompression of signal used in the medical domain. Techniques such as HMM, DCT, as well as PCA are employed for compression and decompression of signals but these
\end{abstract} approaches still possess some disadvantages. Therefore, to overcome these issues, a chip-level design for Artificial Neural Network is proposed that makes use of FinFET $32 \mathrm{~nm}$ technology and includes sigmoid activation function (SAF), Gilbert cell number, as well as bias circuits to prolong the compressed magnitude relation and accuracy. As a result, with the help of the Cadence Virtuoso analog tool, the Artificial Neural Network has been designed using FinFET 32nm technology along with all the details of sub-units such as Layout vs Schematic (LVS), Design rule check (DRC), RC extraction as well as chip level (GDS-II). Feed Forward Artificial Neural Network (FWANN) is considered as one of the most basic types of ANN and it is implemented using the concept of Back Propagation (BP). The simulation results of the suggested 16-bit 6TRAM cell were found to have $8 \%, 21 \%$, and $0.9 \%$ improvement in consuming power, delay, and compressed data losses respectively.
Keywords- ANN, FWANN, SAF, HMM, PCA, SRAM, FinFET

\section{INTRODUCTION}

The science that deals with perception mathematically is named Artificial Intelligence (AI). Artificial neurons are employed by carrying out the artificial Intelligence as genuine $\&$ it is composed of many analog parts. Intelligence is the calculation factor to realize goals within the world. In this study, the execution of neural network construction utilizing a backpropagation formula for data compression and decompression is done. The somatic cell designated contains multiplier factor and adder in conjunction with the tan-sigmoid outcome. However, they achieve instruction design in the analog domain owing to this the full neural design is an analog construction [10]. Practically, the network's instructions are understood with the help of known input as well target along with input. Initially, the load and bias are expected to be constant for a network [1]. The differences among the somatic cell output obtained from 1 and 2 equations along with the target are determined through the learning mechanism. To redesign the load, this fault is used and thereby bias components. This strategy was used until the fault exceeded a set bound that could be tolerated. Analog circuits (AC) are used to represent and execute a neural architecture in analog VLSI equations 1 and 2 [2]. The analog computation of neural networks reduces the circuit overhead and thereby avoiding the conversion of real-time signals into digital signals. The structure of the neural network proposed in this paper consists of a set of input and one output somatic cell that includes three hidden layers of neurons [3]. The Neural architecture is stated as a feed progressive network as well as the learning design is 
employed for BP that is completed within the analog field. As a result, the somatic cell described in this paper can learn for both digital and analog applications. Logic functions like AND, OR, XOR, and NOT gate are implemented using the complex neural model to define Neural Design's digital learning ability. The neural network's analogue functionalities such as inputs of a sine wave, amplification, as well as frequency extension ability, are generated [11]. Whenever the input component intensity matrix is of small magnitude then NA is utilized for compression and decompression. The above-mentioned neural architecture is frequently employed in a variety of analogue signal processing operations. Huge trans conductance electronic devices are used as a junction [4][5] to accomplish the feedforward neural network as well as the feedback learning circuit.

\section{PROPOSED METHODOLOGY OF DESIGN OF GILBERT MULTIPLIER}

The modified Gilbert number, which might be a fourquadrant multiplier, is implemented as a conjunction circuit. The characteristic curve is approximately linear over the small-signal span as well as nonlinear during the large-signal span. The non-linearity, although doesn't cause any non-reliability. We also employ a MOS linear electrical device at the output terminals of the multiplier to convert current to voltage because the Gilbert number comprises current as an output. We usually adjust the Gilbert number to obtain vector extension when performing a dot-product operation. The number's unique output current is combined with two current buses before being converted by linear MOS resistors. This enables the development of larger vector multipliers, such as three- or four-dimensional vector multipliers [13]. A huge Trans-conductance electrical device can be used to generate a Sigmoid function. The trans-conductance, as well as bias current, are identical to each other, and the output will be a tan-1h [14]. Furthermore, the output of the Trans-conductance electronic equipment is current; however, to convert the output into voltage, we usually use five diode-coupled MOS transistors as linear resistors. Two linear feedforward networks are used to examine and handle the input-target signals. The input-output signal is repeatedly applied to the neural network throughout execution and thereby enabling the network learning effectively via converging to a set of maximum weights [15]. Further, the input signals are evaluated using a counterpart of such weights in a separate feed-forward network. There is no necessity to establish an additional circuit for balancing the weight in this proposed model. Due to the elimination of the load interface, the number of required connections is also minimized. The feedforward, as well as learning subcircuit, are the two subcircuits in this chip unit [4]. Two inputs, z, and pi, as well as a pair of outputs, $\mathrm{yj}$, and oi, and also a set of threshold units, I/joules, are included in the architecture of the $6 \times 2$ module chips. The $6 \times 2$ subsystem chip is designed for 1-dimensional '1D', 2-dimensional, '2D MUL', and 7-dimensional vector multiplier factor. There exist 14 capacitors for 12 network loads, wji, as well as a pair of threshold weights. ' $S$ ' suggests that sigmoid performs generator, 'MUX' is associated analog electronic device are proposed [21]. The feed-forward circuit generates a pair of outputs from 6 inputs, a pair of threshold units as well as 14 weights.

Within the learning network, the outputs are given as input to the neighboring layer and are further used to update the network loads, wji, as well as the threshold weights e [8]. The processed loads are then fed to the feed-forward circuit and are later used to generate backpropagation error signals for the previous layers [16]. The desired target signal is furnished within the output layer via the error terminal Ei. The error terminal gi is then used to produce back-propagated error signals. Backpropagation in MLP-ANN is the process of analyzing these signals to obtain an error of the previously hidden layer. The error signals will no longer be transmitted backward if there is no bottom layer. Throughout the training process, these error signals adapt the network loads and threshold weights to avoid interruptions [7]. In a communication system, the conversion of frequency is carried out with the help of Gilbert's MIXER. Therefore, it is essential to design a MIXER for modern engineering applications with low interference, easy implementation, as well as low power. The proposed research work is performed with $45 \mathrm{~nm}$ technologies by taking into account the significance of $45 \mathrm{~nm}$ technologies over $90 \mathrm{~nm}$ and $65 \mathrm{~nm}$ technologies respectively [9]. This research work presents a feasibility study area for layout sketch of Gilbert cell using VLSI technology. In VLSI design for mobile applications, power consumption might be a controlling factor. The resulting power consumption regulates the VLSI chip's packing and manufacturing. One of the costeffective ways to reduce power consumption in modern electronic systems is by reducing dynamic power dissipation in synchronous digital computer circuits [5]. Since supply voltage falls as the technology advances, all power and ground-level fluctuations in the power supply have an impact on the analogue circuit's output [6]. Various layouts, easy and accessible process, logic circuits, CMOS circuits, as well as physical design are some of the basic features of VLSI technology. To compress the image, the network structure is used which 
is introduced and illustrated in the preceding section. The image is composed of picture element brightness is fed to the network for compression and reconstruction [17-13]. The 2:3:1 somatic cell projected a basic capacity of constricting the inputs, since there are 2 inputs, and one output compression established is $50 \%$. Analog to digital converters is not required since the input given to the network is in analog form. One of the most important accomplishments of this research work is the modeling of a 1:3:2 neural network for reconstruction functions. The hidden layer of the neural network contains three neurons, whereas the output layer has two. The back Propagation algorithm is the training method used in this network. Once the network has been implemented with varied inputs, the error circulates from the reconstruction block to the compression block, and the two different methods used are discrete and can be utilized as compression blocks as well as decompression blocks separately. The Artificial Neural Network (ANN) is an AI expectation calculation that is enlivened by the sensory system. In ongoing writing, ANN is utilized to see errors in FinFET transistors condition [6].

$\mathrm{X}_{0}, \mathrm{X}_{1}, \mathrm{X} 2, \mathrm{X} 3 \ldots \mathrm{X}_{\mathrm{n}}$ and $\mathrm{W}_{0}, \mathrm{~W} 1, \mathrm{~W} 2, \mathrm{~W} 3 \ldots \mathrm{W}_{\mathrm{n}}$ are the inputs and weights of the neuron then,

$\mathrm{F}(\mathrm{X}) \quad$ is
$\left.\mathrm{X}_{1} \mathrm{~W}_{1}, \mathrm{X}_{2} \mathrm{~W}_{2}, \mathrm{X}_{3} \mathrm{~W}_{3} \ldots \mathrm{X}_{\mathrm{n}} \mathrm{W}_{\mathrm{n}}\right)=\mathrm{f}\left(\sum_{i=0}^{n} X_{i} W_{i}\right)$

$\mathrm{f}\left(\mathrm{X}_{0} \mathrm{~W}_{0}\right.$

To start with, comprehend to review how an ANN functions, consider a solitary neuron model as appeared in Fig.1. The component of the data test is $n-1$ as $\mathrm{X} 0$ is the one-sided input. The weights ' $\theta$ ' is introduced to little \pm values fixed on 0 . At this point when the ndimensional data (counting the predisposition input) is sent through the ANN, it is at first increased by its underlying weights [24]. When the weighted total of the $\mathrm{n}$ measurements surpass edge esteem than the neuron fires drifting point esteem which is then gone through the actuation work $f(\theta \mathrm{T} X)$ for mapping the incentive to give a yield. At this point, when Cost Function is high, Backpropagation calculation is utilized in changing loads of the ANN to have ease. In the following cycle, the next information is nourished and while the loads are refreshed. The above procedure is recursively pursued until we have easy work. Different such neurons associated together are known as a Neural Network. The essential activity of handling the contributions to predicting the expected output from a single neuron as shown in Fig.1, makes the ANN plan simple to actualize in VLSI.

\section{PROPOSED DESIGN METHODOLOGY FOR A NEURAL NETWORK WITH ADAPTIVE FILTERING}

ANN design using analog circuits is the major challenging for fabrication of integrated circuits to optimize the power and delay for high throughput, in [6] ANN is designed in Cadence platform and transistor size is considered based $\mathrm{I}_{\mathrm{dc}}$ but its also depending on the threshold voltage $\left(\mathrm{V}_{\mathrm{t}}\right)$. Compared to [6], in this work, the transistor size is dynamic i.e based variations of $I_{d c}$, $\mathrm{V}_{\mathrm{t}}$ and oxide thickness, width to length ratio is defined so that overall performance is improved.

In this work, four phases are having different input widths as input data to $\mathrm{ANN}$, and the same data is processed through Gilbert cell multiplier, a single neuron, and activation function for compression of the data into single data as shown in Fig.6.1. The network modeling and its performance employing a neural chip are the main blocks of ANN. The compressed single data is input to the decompression for the reconstruction of the original data as shown in Fig.6.15. The proposed model network consists of input, output, and hidden layers along with signals. There are many sorts of adjustable filtering for neural network applications that have been considered: one is Network modeling: A network of hidden fabric consists of easily identifiable input and output signals in various application areas. The application of input is used to identify an appropriate neural network and its output as the neural network's target value is used as one of the methods for obtaining information regarding the hidden system's optimal solution. The neural network produces feedback that is similar to the hidden networks. We focus on providing a sine wave to an unconventional circuit in a basic example, which results in a deformed circular function wave shape. Forecast: Neural networks will evaluate the upcoming values from the existing previous signals of input. The actual value is the available input signals, here the neural network receives deferred system input signals as an input. To forecast the existing input signal, the neural network functions should strive for no error.Fig.2 depicts the design and validations of the model using ANN chip, the sine wave is applied to find the unknown data to the circuit and it has resulted in an error-free sine wave. The uncertain system's output is fed into the ANN chip's signal which is then used to evaluate and rectify error signals that are generated by the processing unit.

Prediction: ANN system can assess future qualities from existing as well as previous information signals. The objective is to bestow data signals as well as postponed signal information which fills in as a 
contribution to the neural system. The neural system mistake toward zero. attempts to see the present data signal to drive the

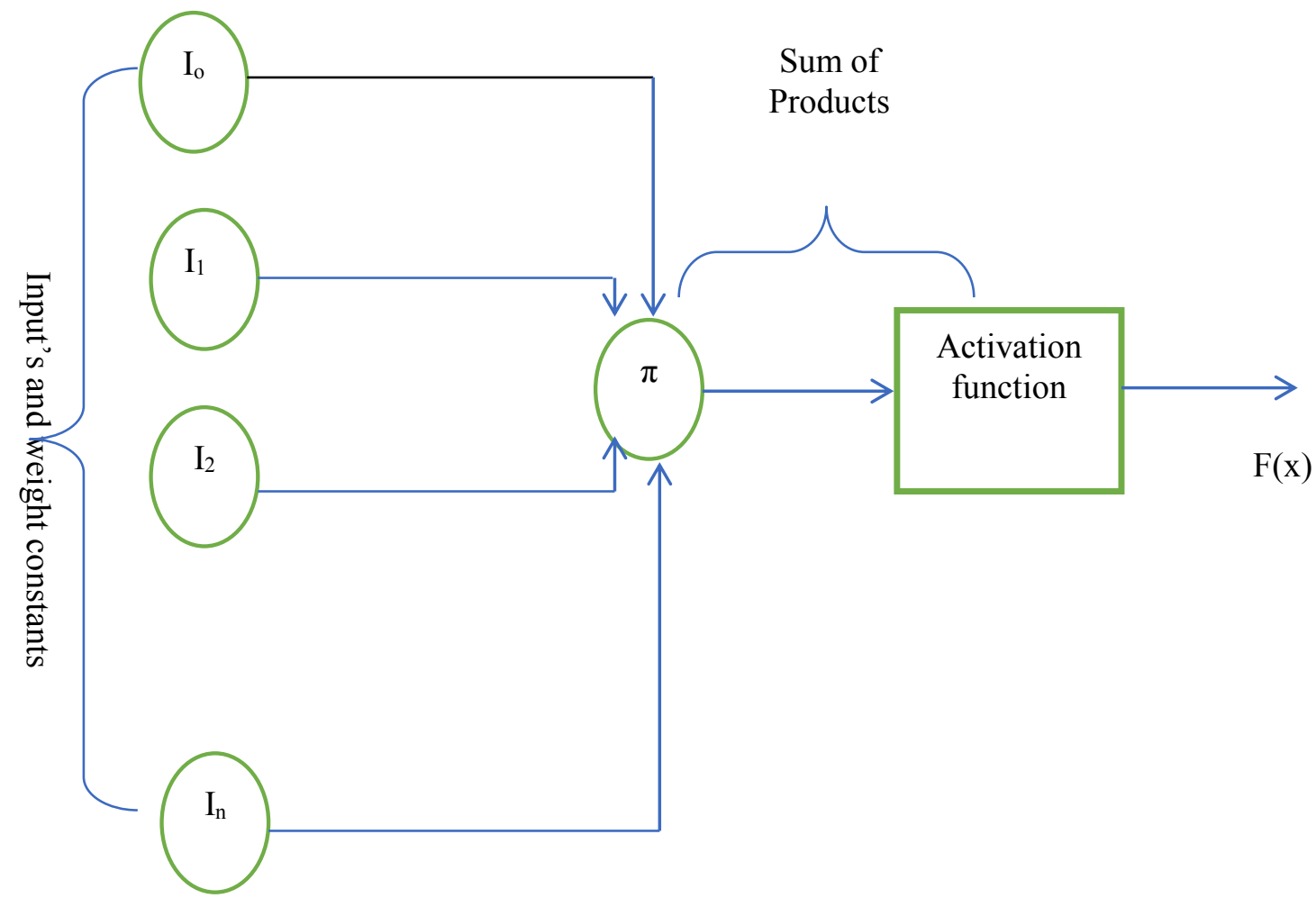

Fig.1. Single neuron and its mathematical model

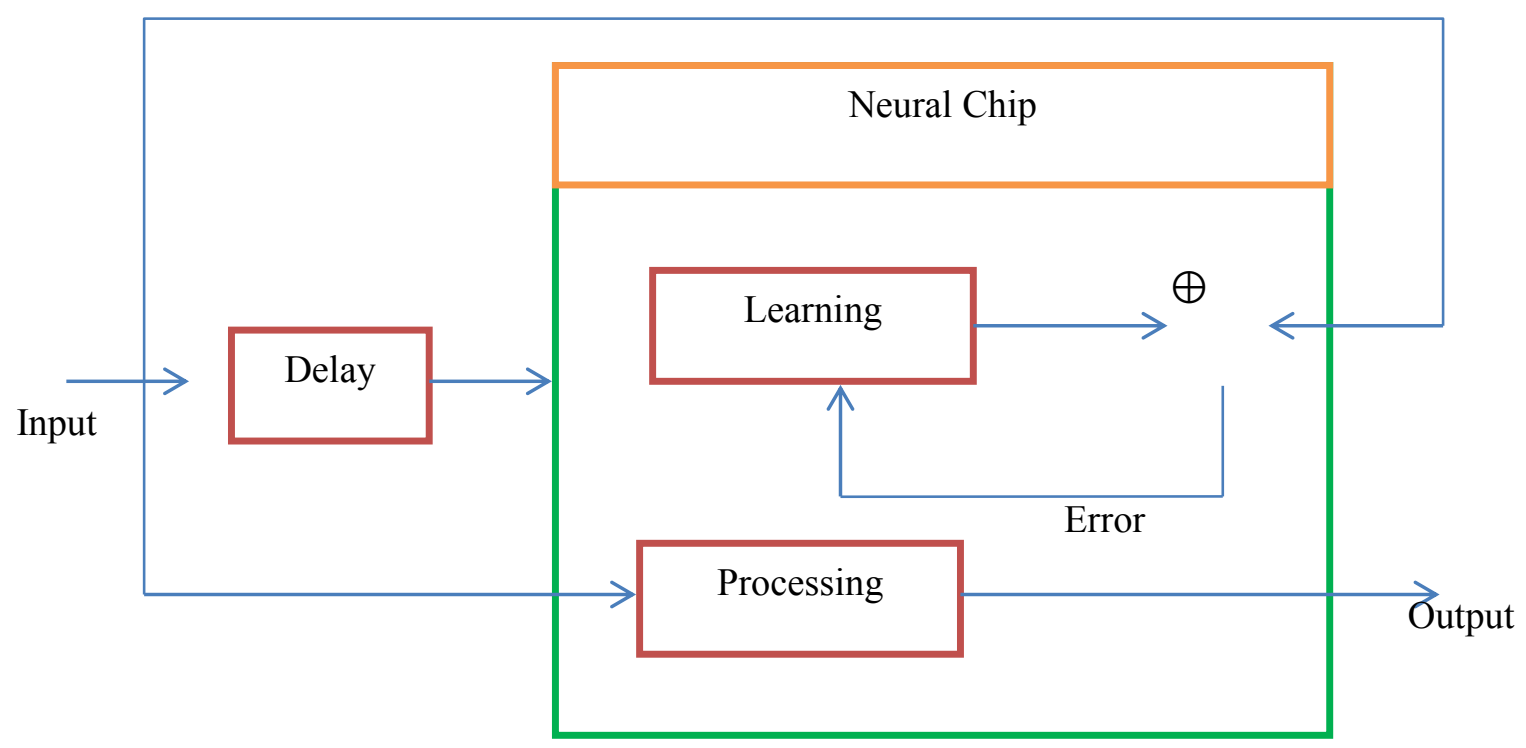

Fig.2. Design of prediction for error while training the network [6]

The prediction for error calculation appeared in Fig. 1 and the outcome has appeared in Fig.3. The output of the neural chip's processing unit must be placed in front of the input signals in this research work. The simulation and experimental results show the effective prediction ability of the neural chip. Decompression of the signal: In this application, the neural system activities to reconstruct the original data and its sign, which is accepted to have been changed by adding weights of each neuron in the architecture. The response 
to the neurological system is the output of the hidden plant. The accurate approximation of the neural network is the delayed unknown plant's information. The learning computation reduces a slight number of errors and thereby enabling the neural networks to construct a backward model of the intriguing plant. The output of the neural chip's processing module must be an effectively delayed counterpart of the input signals as shown in Fig.2. The neural chip's output is transformed into a data signal in the form of a sine waveform as demonstrated in Figure.4.

The output of the neural chip's process unit must be previously known to the input in this example, as well as the investigational results illustrate the exceptional predicting capacity of the CRF neural chip.

Counter modeling: The neural network tries to obtain a delayed form of the signal that is expected to have altered unknown Plant with additive fault throughout this execution. The unrecognized plant's output is fed as input to the neural network with the backward unidentified input data serves as an expected value for the neural network. As a result, learning model code introduces a slight inaccuracy and enables the neural network to extend the countermodel of unidentified plants. The output of the neural chip's process unit is shown in Figure 4 as a substantially degraded form of the input. The output of the neural chip is used as the input in the sine wave simulation.

Feed-forward ANNs with learning to be used to accommodate the signal process. A feed-forward network that comprises learning circuits, as well as a reliable network that processes the replica of weights, is included in a typical chip. The results demonstrate that the learning circuits will present the input-target temporal signals when the networks along with learning circuits are evaluated and verified. The proposed approach scales effectively from three to huge networks and is suitable for large-scale adaptable signal processing. Using Virtuoso, the circuits are implemented and the observational data demonstrate their efficient functioning.

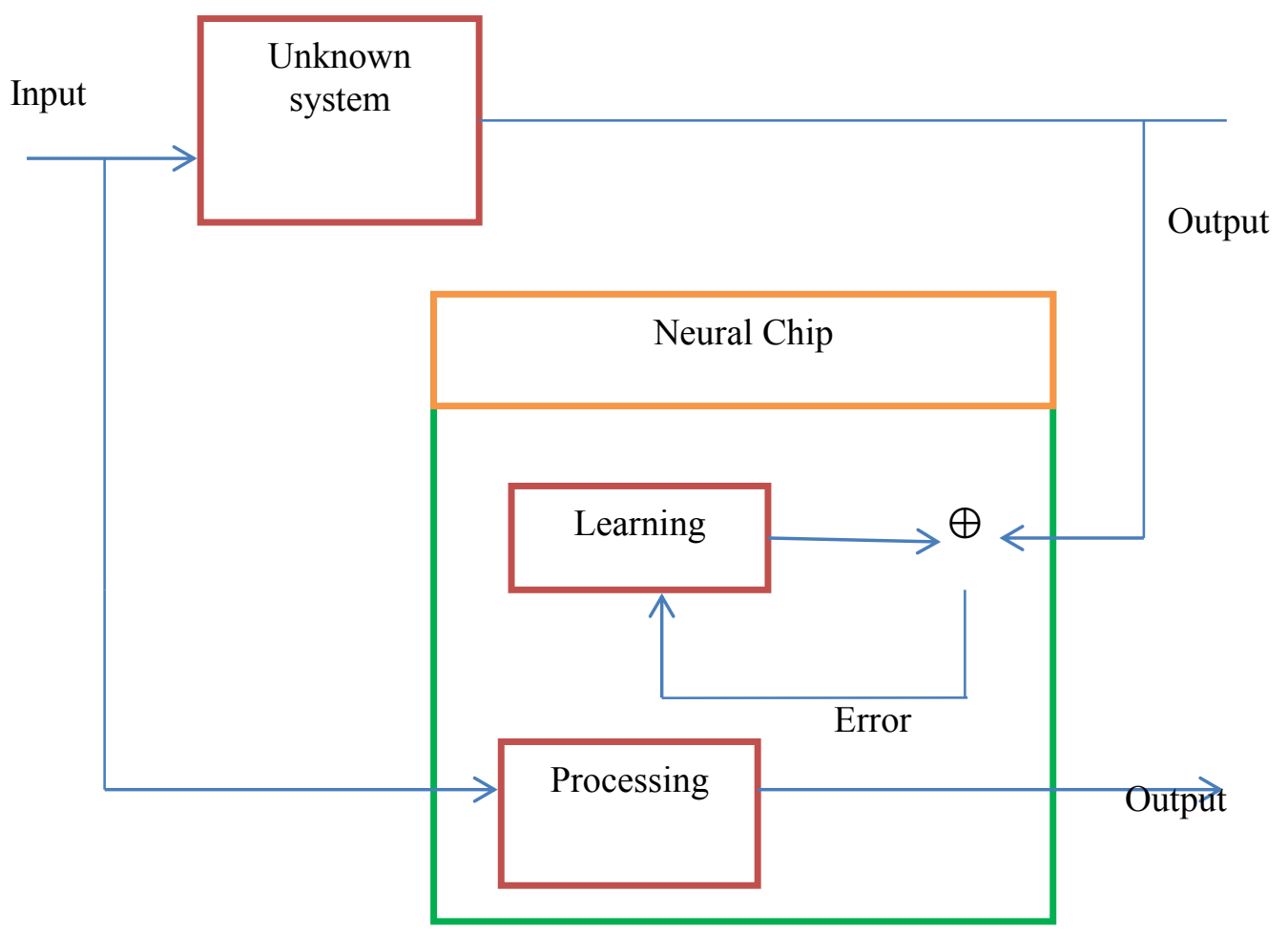

Fig.3. Design of modeling of the system for a number of iterations [6] 


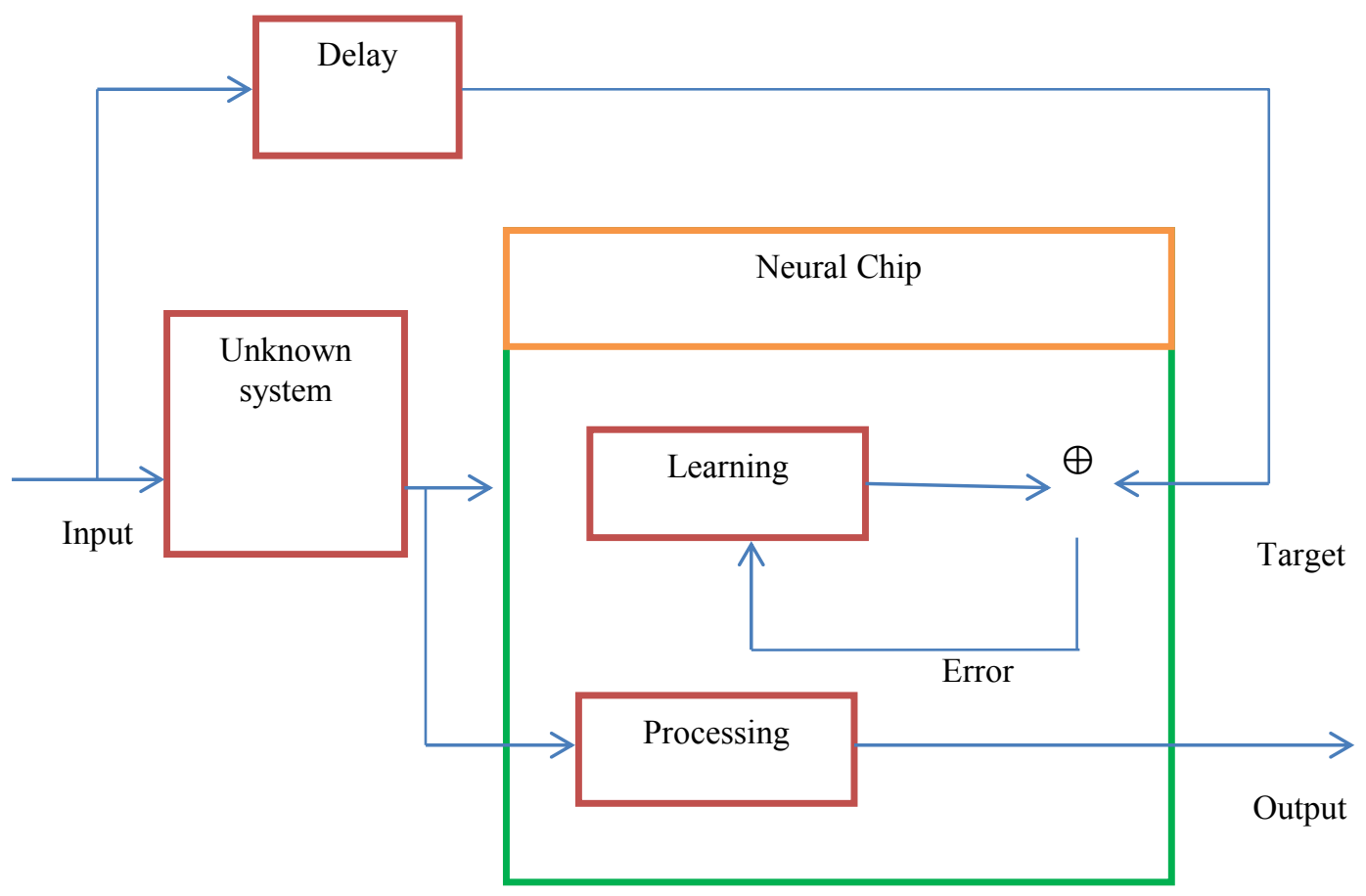

Fig.4. Design of Inverse Modeling to monitor the target output [6]

A revised learning guild line is used to execute standard Weight Update:

$>$ For every load conjunction, it multiplies its output delta in eq. 1 and inputs refreshing to induce the slope of the load.

$>$ Bring the load in another way of the slope by removing a quantitative relation of it from the weight. This quantitative relation affects the speed and standard of learning, and it is referred to as learning to rate. The indication of the gradient of a load indicates wherever the error is increasing, that's why the load should be updated within the other way. These phases go on replicating till the execution of the network is acceptable.

Fig.1 depicts the neural network, During this network, inputs $\mathrm{v}_{1}, \mathrm{v}_{2}$ (where is $\mathrm{v} 1 \mathrm{v} 2$ are used as inputs) concerns with the load matrix, then this load inputs of the adder are added up. The outcome produced by adder blocks is given to the nerve cell Activation outcome. The outcome of the activation task is increased by the average once more, and given to the input structure of the output layer. This layered design of the neural network is enforced in VLSI for analog parts. Gilbert cell multiplier factor, summer, and differential amplifier are used for various blocks. The performance of neural parameters with on-chip learning for generalized signal processing applications in analogue VLSI is the primary goal of our research work. In this chapter, the analog parts like the Gilbert Cell multiplier factor (GCM), nerve cell activation function (NAF) are used to perform neural specification. The analog part used contains multipliers and summers beside the tan-sigmoid function circuit utilizing MOS semiconductor in the subthreshold region. The above-mentioned neural architecture is given training in the analogue domain by using the backpropagation (BP) rule as well as the most recent load storage technique. Layout sketch and authentication of the proposed model are analyzed using the Cadence analog 1.6.4 tool. The technology utilized in planning the layouts is Virtuoso $0.5 \mathrm{u}$, Tight Metal. Typically, the desired process power (or, in alternative words, the intelligence) of those applications is the propulsion for the quick development of this field. This stratified structure of neural networks is enforced in VLSI utilizing analog elements. Gilbert cell multiplier factor, summer, and differential electronic equipment are used for various blocks.

To scale back, the facility demands we have designed the neural network utilizing advanced technology. The human brain is composed of neurons that send activation signals for every alternative thereby making intelligent thoughts. A neural network in its iterative version is also termed an artificial neural network. This network comprises neurons that can transmit an activation signal 
to another network. The virtual or artificial neural network, at last, can assess the effects of numerous inputs and outputs. As a result, neural networks are often used for different information mining tasks, among that are categorization, pictorial designing, assembling, task estimation, and statistic forecast. In the image process, neural networks are normally used. The projected neural design is capable of executing behavior like wave learning, amplification, and frequency multiplication and might even be used for analog signal process tasks. Nowadays, among multi-GHz communication systems such as wireless devices, optical data lines, microchips as well as ASIC/SOC prototypes, power is considered as one of the most important concepts of model convergence. In a communication system, the conversion of frequency is carried out with the help of Gilbert's MIXER. Therefore, it is essential to design a MIXER for modern engineering applications with low interference, easy implementation, as well as low power.

Further, the rationalized aspect of the VLSI technology is composed of various characterization, generalization of model, logic circuits, Complementary Metal Oxide Semiconductor circuits as well as physical layout. The Gilbert Mixer (cell) is employed as a number block; the major constituent was numerical couple transistors and current reflector circuit. In this work, the Gilbert cell serves as multiplier and summer blocks of the Neural Network. A single-balanced mixer has a unique RF signal. Since the LO signal is more sensitive to interference, this design is extensively employed but LO-IF feedthrough is one of its drawbacks that is if the IF frequency is not lower than the LO frequency, the LO signal may discharge into it. The mixer's low pass filter ensures that the LO signal is not appropriately suppressed and on the other hand the IF signal remains unaltered. To prevent the LO signal from approaching the output, double-balanced mixers are often utilized. Single-balanced circuits that include RF transistors which are connected in parallel as well as the switch pair linked in anti-parallel are essential. As a result, the LO values are added up to zero by doubling the RF signal within the output. This configuration gives a significant level of LO-IF isolation and thereby minimizing the need for output filtering. When compared to single balanced mixers, the doublebalanced mixers are less sensitive to interference because of the differential RF signal. A double balanced mixer is widely known as the Gilbert Cell Mixer and is illustrated in Fig.4. Frequency conversions are performed using mixers, which multiply the RF frequency with the LO frequency to convert it to the IF frequency.

\section{Results and Discussion}

A feed-forward neural system that maps clusters of resilience values that are obtained with the help of wavelet sub-band coding is termed a multilayer perceptron. This vitality esteems are bolstered to the information layer and duplicated with initial loads as given by $\mathrm{Eq}$ (6.1). The back-propagation is the allinclusive rendition of straight perceptron considering its utilization of two concealed layers with nonlinear initiation work by the direct perception. The backpropagation in the neural system is the broadly connected learning calculation for multilayer perceptron. The back-propagation utilizes angle variation for limiting the squared mistake between the system output esteem and the ideal output esteem. The obtained error sign from $\mathrm{BP}$ is cast to compute the weight refreshes which speak to the intensity of information obtained on the system. Fig.5. portrays the engineering of MLP design. In back-propagation, the loads are refreshed after each example and by taking one example $\mathrm{m}$ at once as pursued:

\section{Forward Phase:}

The pattern $X_{j}^{(l)}$ is applied to the input layer and further transmits the data via the network till the computation of ultimate outputs has been done for each $\mathrm{i}$ and $\mathrm{L}$.

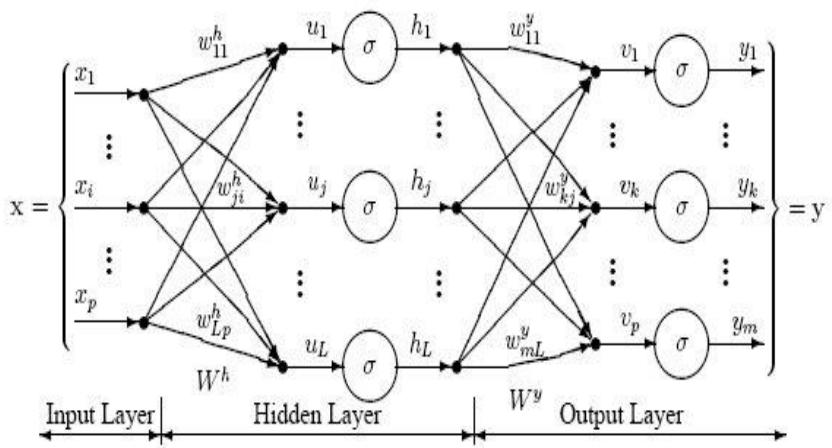

Fig.5. Multilayer Perceptron architecture

$$
X_{j}^{(1)}=\theta\left(s_{j}^{(1)}\right)=\theta\left(\sum_{i=0}^{D(L-1)} x_{j}^{(1)} w_{j}^{(1)}+w_{j}^{(1)}\right)
$$

Here..the total neurons present in the layer (L-1) is indicated by $D(L-1)$, the output of the $j^{\text {th }}$ neuron in the $(1-1)^{\text {th }}$ is specified by $X_{i}^{(l-1)}$, synaptic weight confined in the existing neuron is given by $\mathrm{W}_{\mathrm{ij}}$ (i), present bias weight of neuron is represented by $\mathrm{W}_{\mathrm{ij}}$ (i), and the output of an existing neuron is indicated by $X_{j}^{(l)}$.

Back Propagation: The error slope drop vector is used to update the weights and inclinations. A system yield vector is acquired after the utilization of the data vector 
during the forward calculation stage. An objective vector is given to the system, to drive the output of the system towards, the normal objective worth. Starting with the output layer and stepping back to the data layer, the standard errors, as well as slope, are computed as follows:

$$
e_{j}^{(1)}=\left\{\begin{array}{llc}
\left(u-x_{j}^{(1)}\right) & \text { for } & l=L \\
\sum_{w}(1+1) s_{j}(1+1) & \text { for } & 1=1,2,3, \ldots \ldots . . L-1
\end{array}\right.
$$

(2)

where $e_{j}^{l}$ is the error term for $\mathrm{j}^{\text {th }}$ neuron in the $1^{\text {th }}$

layer $\mathrm{s}_{\mathrm{j}}^{(\mathrm{l}=1)}=\mathrm{e}_{\mathrm{j}}^{(\mathrm{l}+1)} \theta\left(\mathrm{s}_{\mathrm{j}}^{(\mathrm{l})}\right) \quad$ for $\quad 1=1,2, \ldots$,

where $\theta\left(s_{j}^{(l)}\right)$ is the derivative of the activation function.

calculate the changes for all the weights are calculated as follows:

discovering any errors utilizing the mean square mistake (MSE) and it is given by 5 .

$M S E=\sum_{i} \operatorname{error}\left(d^{i}-y^{i}\right)=\sum_{i}\left\|d^{i}-y^{i}\right\|^{2}$

The error can be reduced by passing error from output to the hidden layer and then to the input layer using backpropagation ANN and at the input layer, the weights are updated. $n=i 1{ }^{*} 1+i 2 * w 2$ (1). The Fig.6. shows the transistors level schematic diagram for a
$\Delta \mathrm{w}_{\mathrm{ij}}^{(l)}=\eta \mathrm{s}_{\mathrm{j}}^{(1)} \mathrm{x}_{\mathrm{j}}^{(1-1)} \ldots \ldots . . .1 .=1,2, \ldots \ldots . \mathrm{L}$

Here the learning rate is given by $\eta$. Update all the weights

as

follows: $\mathrm{w}_{\mathrm{ij}}^{(\mathrm{l})}(\mathrm{L}+1)=\mathrm{w}_{\mathrm{ij}}^{(\mathrm{l})}(\mathrm{L})+\Delta \mathrm{w}_{\mathrm{ij}}^{(\mathrm{l})}(\mathrm{L})$

Here $1=1,2 \ldots \mathrm{L}^{(1)}$ and $\mathrm{j}=0,1 \ldots \mathrm{L}^{(\mathrm{l}-1)}, \mathrm{W}_{\mathrm{ij}}{ }^{(1)}(\mathrm{L})$ is the existing synaptic weight.

The refreshed synaptic weights $\mathrm{W}_{\mathrm{ij}}{ }^{(1)}(\mathrm{L}+1)$ are used for the consequent feed-forward cycle. It is the full cycle of the period; the term time frame is utilized in neural systems preparing for depicting a total go through all the preparation designs. The neural net's weight could be renewed after every sample that is shown to it, or merely once after the phase. The total design is prepared for the arrangement of selected images from the database into typical or unusual. Multilayer perceptron design has been prepared in the system, a forward way from the information layer to the output layer. This output is contrasted with objective reference esteems with single neuron and its layout design and its DRC results using Cadence

Virtuoso tool by using $32 \mathrm{~nm}$ technology which is based on FinFET transistors. Fig.7 shows the design for a single neuron using FinFET technology contains five transistors of length $\mathrm{L}=45 \mathrm{~nm}$ and width $\mathrm{W}=240 \mathrm{~nm}$. The designed circuit is simulated in the FinFET package library to verify the compression of data; it is found that almost $12 \%$ of data is compressed. Single neuron schematic cell is converted into layouts and validated using DRC error as shown in Fig.6.

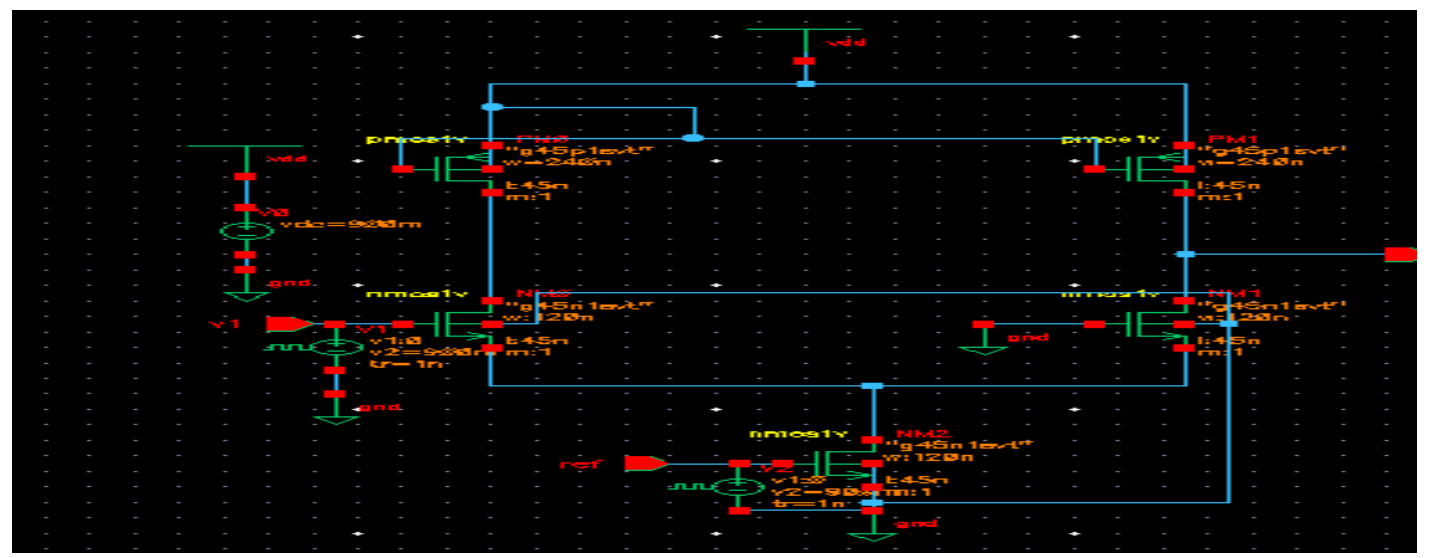

Fig.6. Schematic circuit of single neuron and layout diagram and its DRC results 


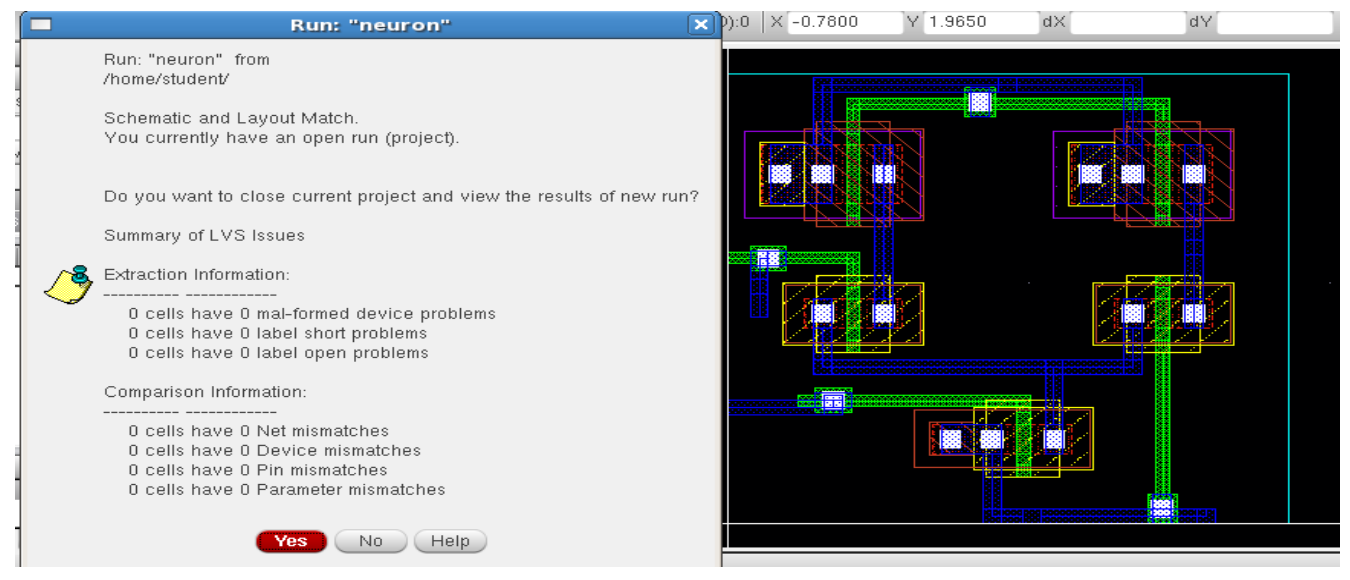

Fig.7. Schematic circuit of single neuron and layout diagram and its DRC results

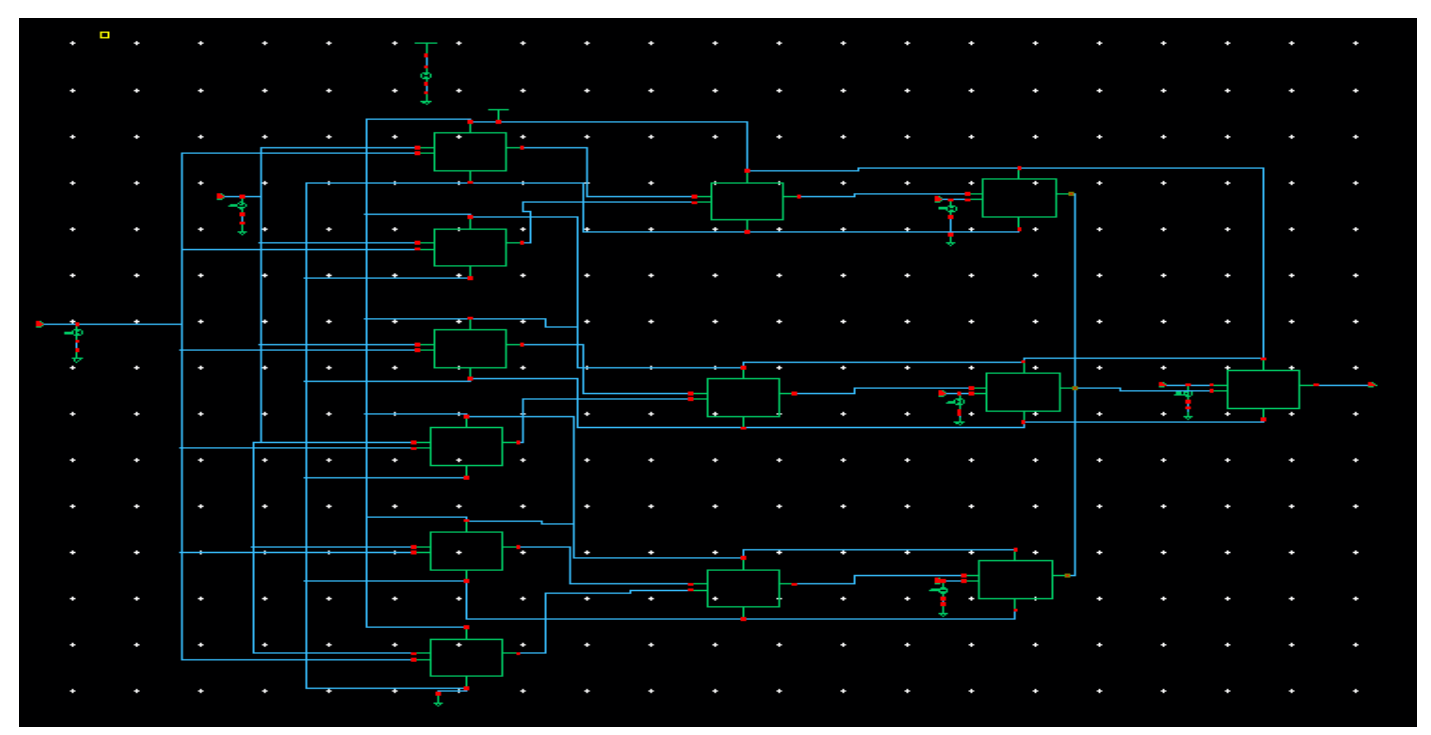

Fig. 8. Block-level design of signal compression using ANN in Virtuoso tool 


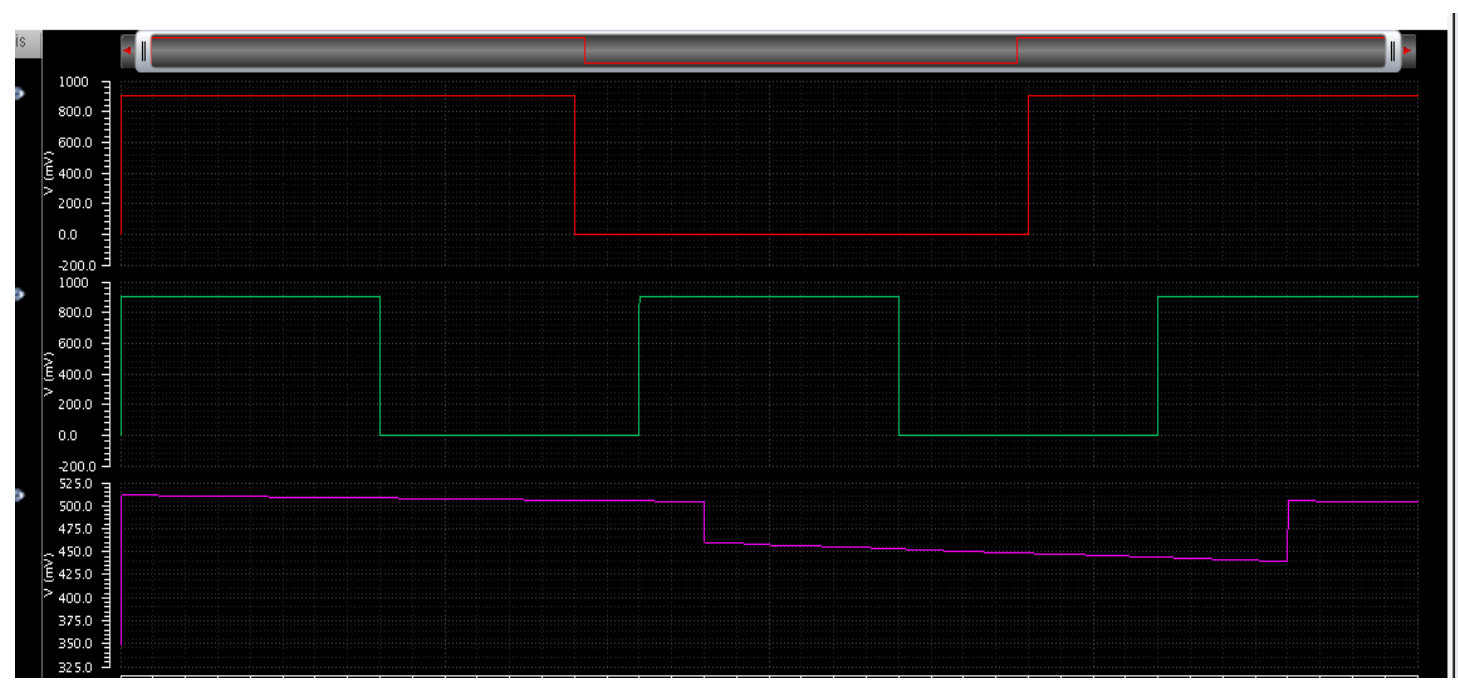

Fig.9. Simulated result of Signal compression using ANN

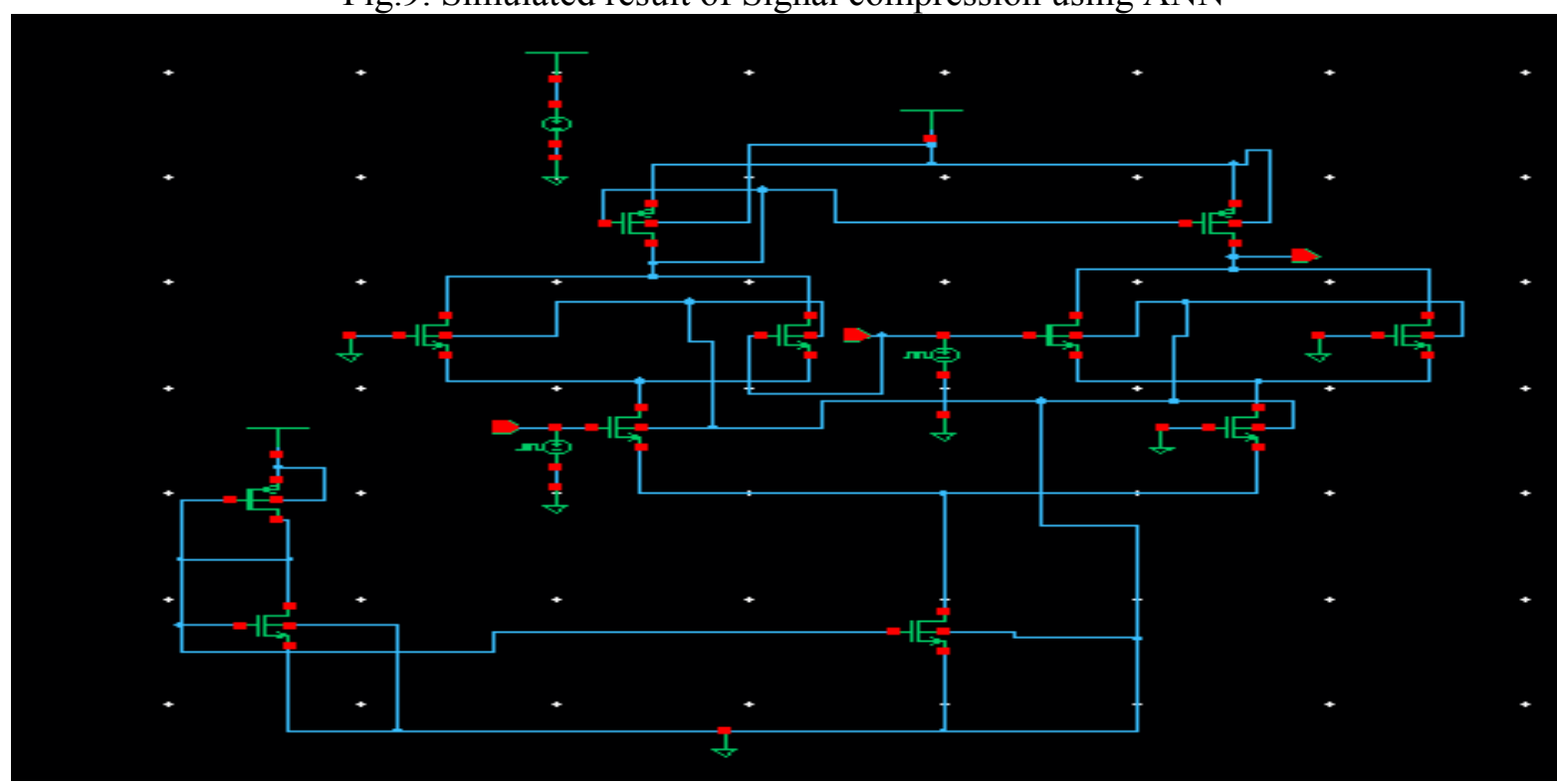

Fig.10. Schematic circuit of Gilbert cell multiplier factor and its simulated results

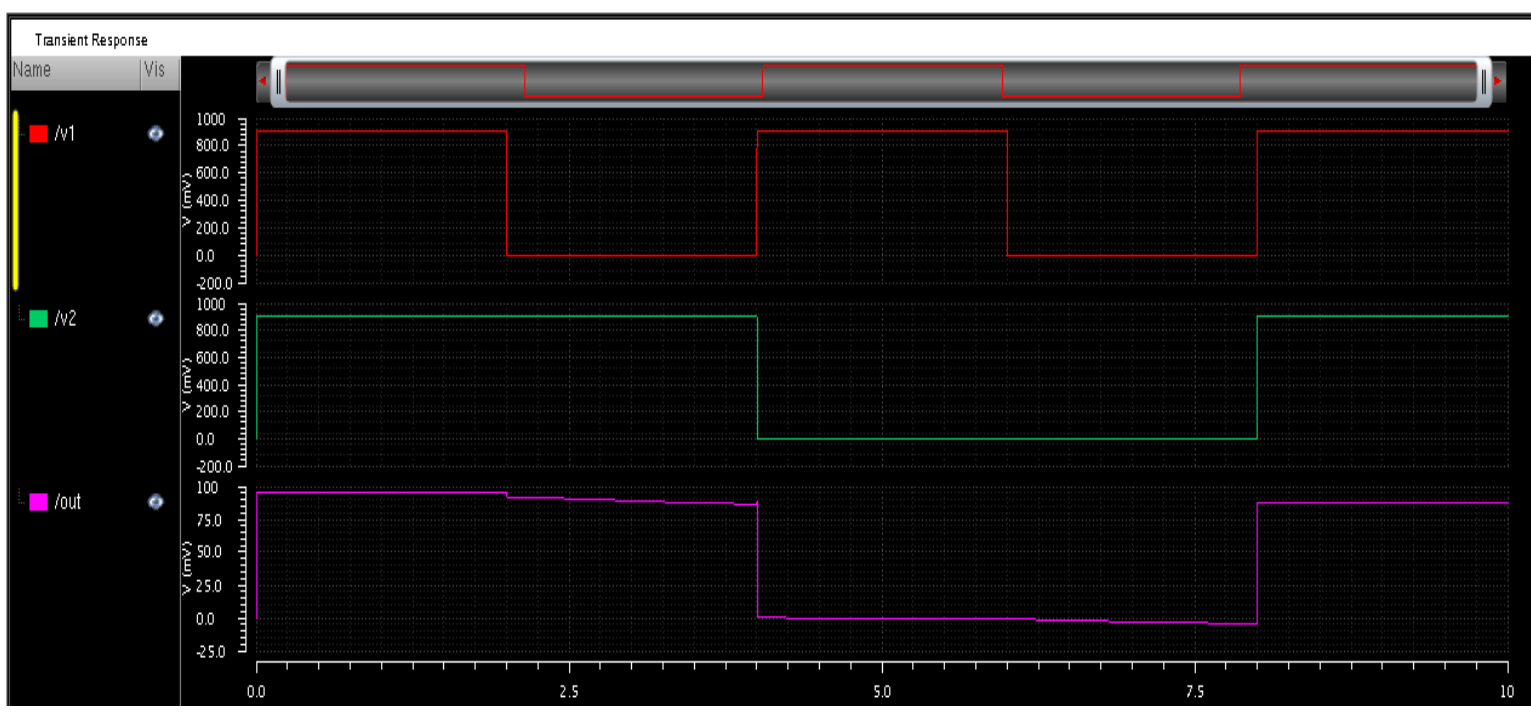

Fig.11. Schematic circuit of Gilbert cell multiplier factor and its simulated results 


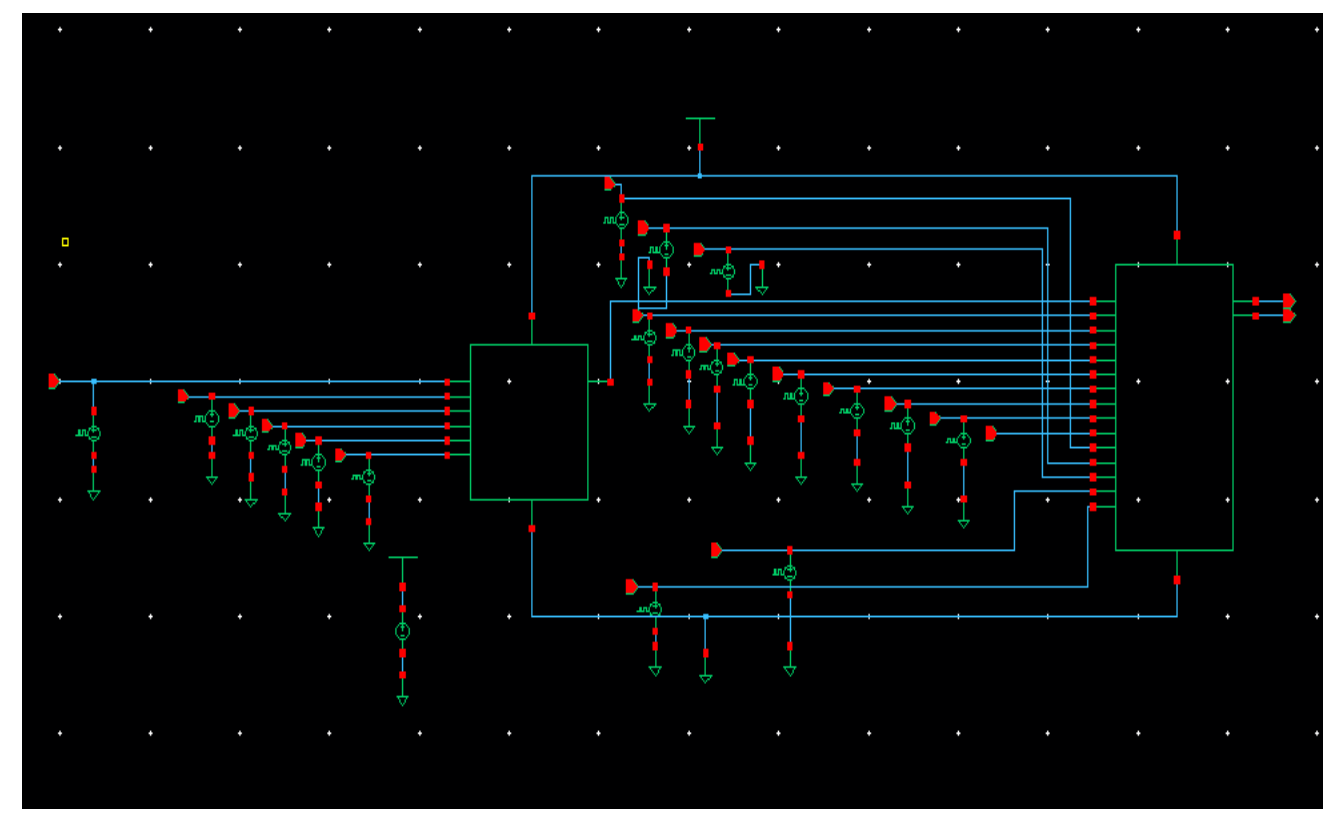

Fig. 12. Top module schematic design with test bench input signals.

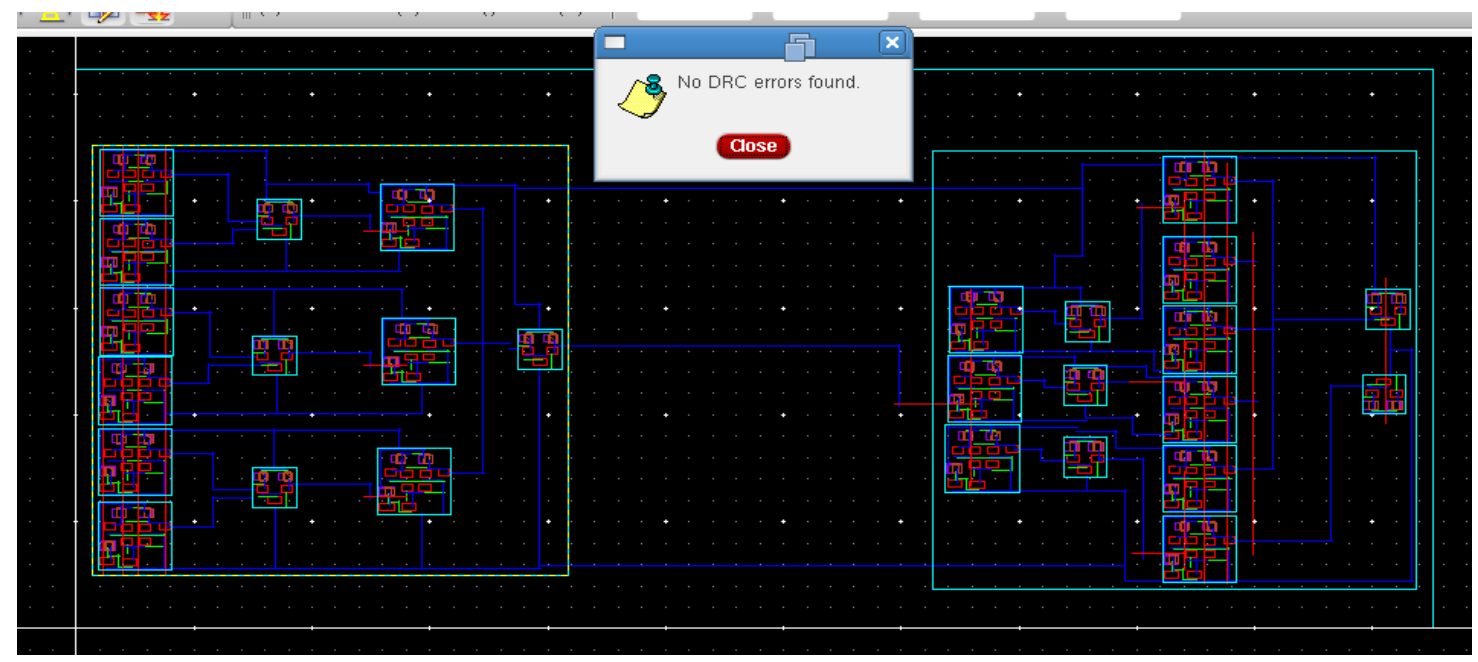

Fig.13. Compression top module layout design with DRC results.

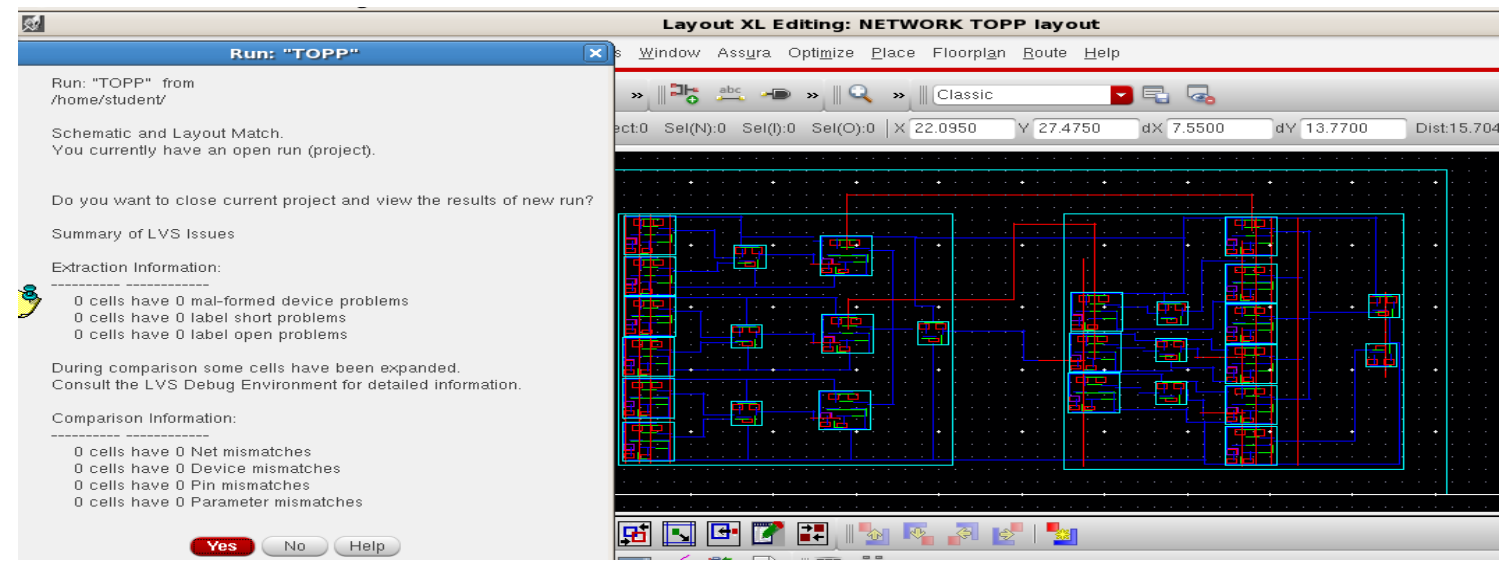

Fig. 14. Top module layout design with LVS results. 


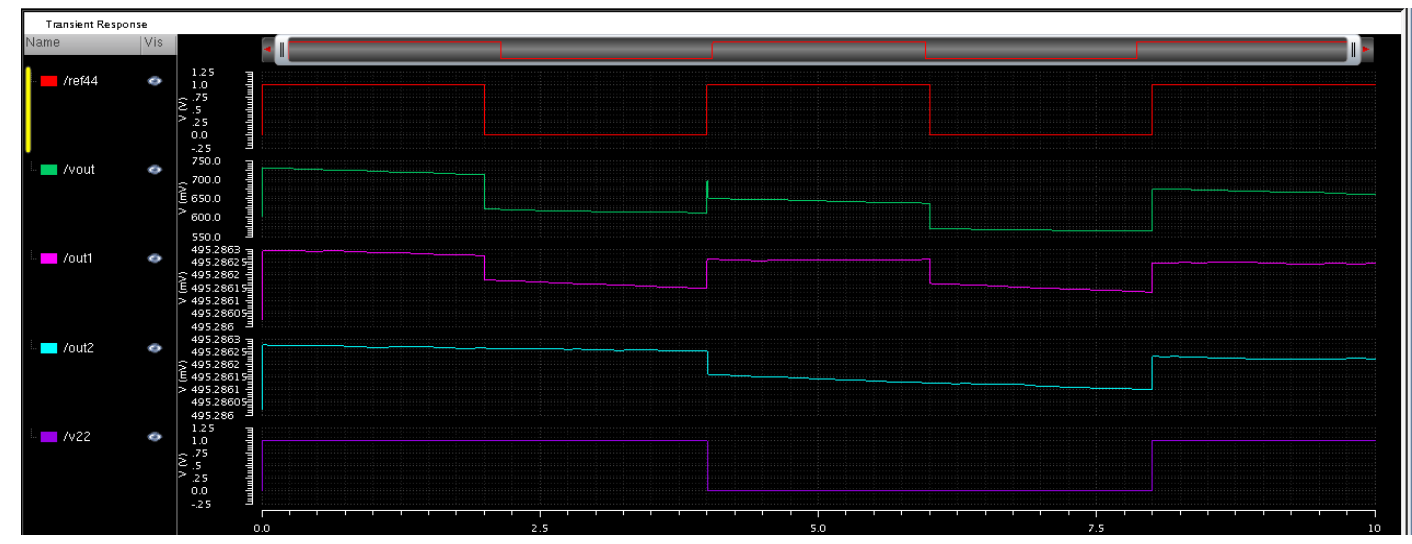

Fig.15. Simulated results of top module for signal compression.

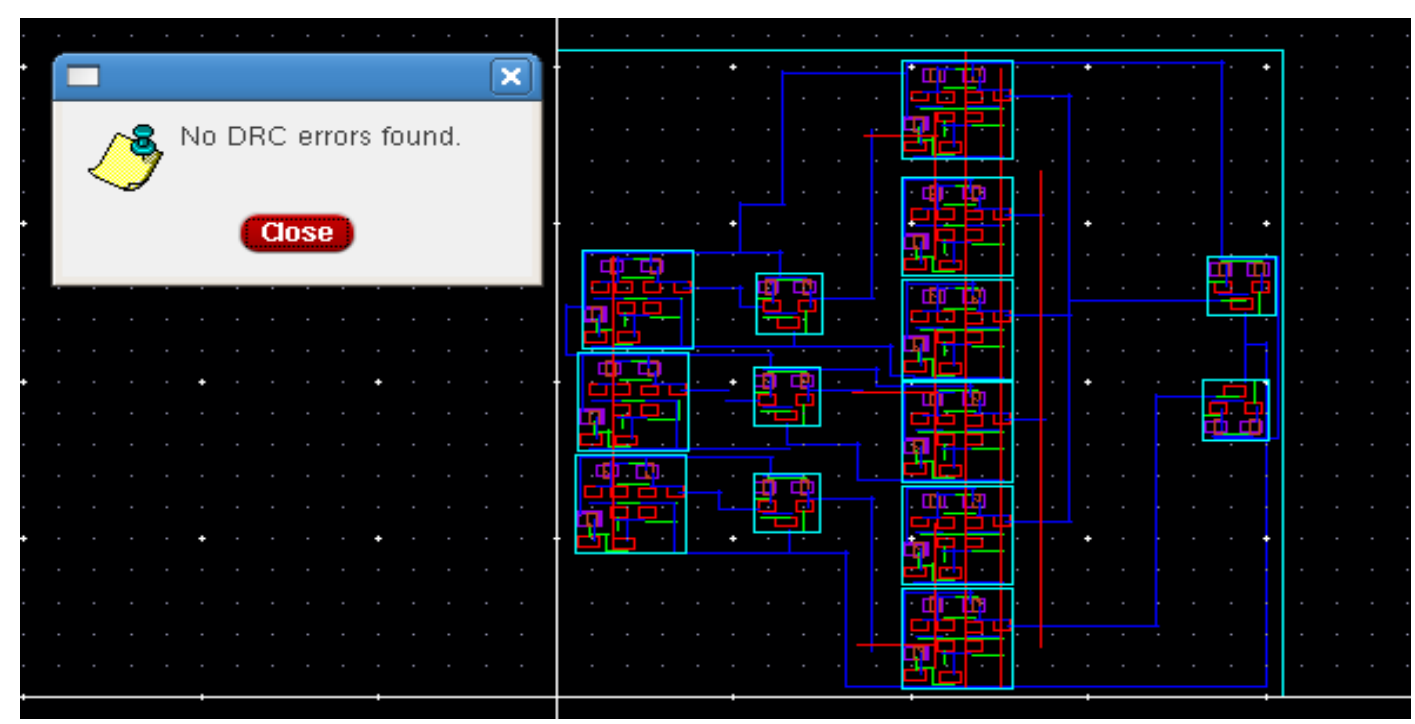

Fig.16. Decompression top module layout design with DRC results.

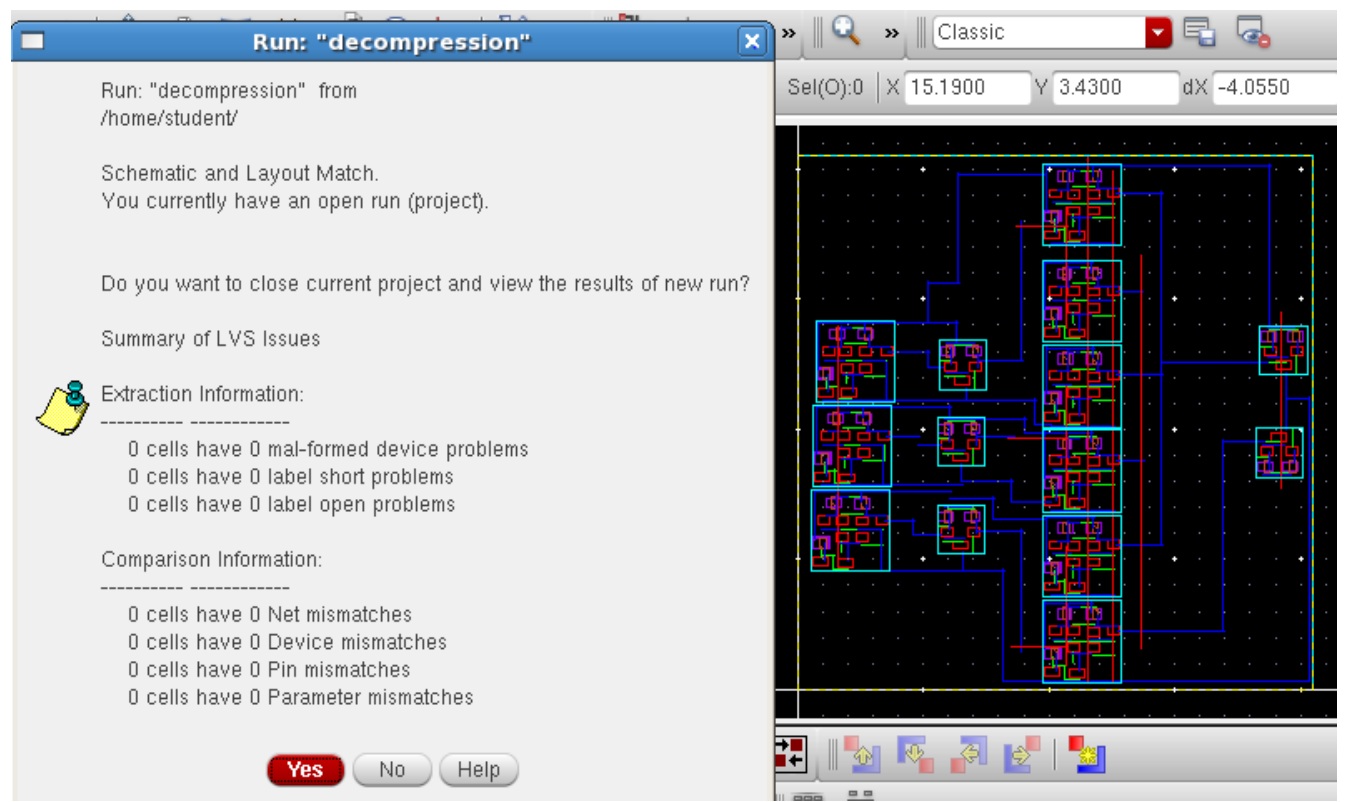

Fig. 17. Decompression top module layout design with LVS results. 


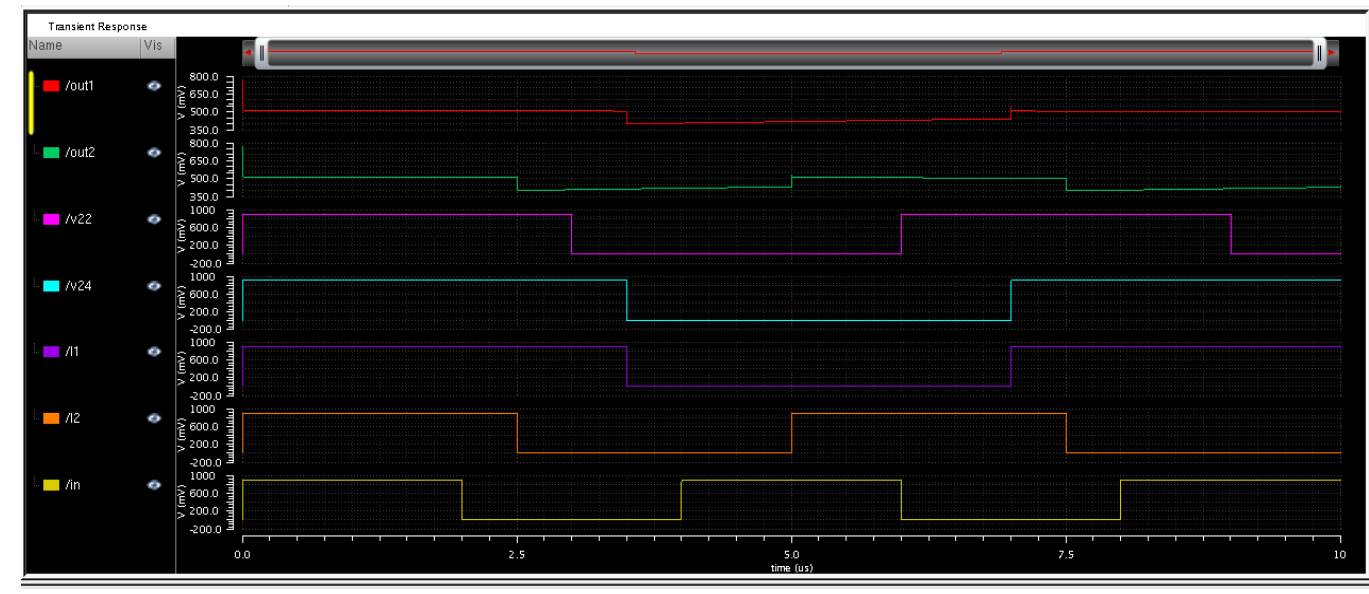

Fig.18. Simulated results of top module for signal decompression.

Fig.8 shows the block level schematic diagram for compression using the Cadence Virtuoso tool by using $32 \mathrm{~nm}$ technology which is based on FinFET transistors. This design is simulated and obtained results are as shown in Fig.9. The proposed design for data compression and decompression using ANN is successfully designed and simulated in the Virtuoso Cadence tool, the obtained compressed and decompressed its DRC are shown in Fig.9 to Fig.18. The pulse single data was provided to the system as a heartbeat wave with v1 linked to $0.5 \mathrm{Vpp}$ along with 50 $\mathrm{kHz}$ frequency, and $\mathrm{v} 2$ to linked to ground. The process was initiated to generate a sine wave with the same periodicity and adequacy as the previous one. As can be found in Fig. 6 this yields the objective connected for the circuit for learning. In this manner, the system figured out how to synthesize the info sine wave as a yield. The system was built for a recurring sine wave with $10 \mathrm{MHz}$ frequency as well as $0.5 \mathrm{~V}$.pp amplitude and is illustrated in Figure.10.The system consistently produced the desired frequency of $10 \mathrm{MHz}$. The output range is between $+.5 \mathrm{~V}$ to $-.5 \mathrm{~V}$. one percent adequacy error, the union time estimated was found to be $200 \mathrm{~ns}$ for $10 \mathrm{MHz}$. Fig.8 shows the block level schematic diagram for compression using the Cadence Virtuoso tool by using $32 \mathrm{~nm}$ technology which is based on FinFET transistors. This design is simulated and obtained results are as shown in Fig.9. The proposed design for data compression and decompression using ANN is successfully designed and simulated in the Virtuoso Cadence tool, the obtained compressed and decompressed its DRC are shown in Fig.9 to Fig.18. The pulse single data was provided to the system as a heartbeat wave with $\mathrm{v} 1$ linked to $0.5 \mathrm{Vpp}$ along with 50 $\mathrm{kHz}$ frequency, and $\mathrm{v} 2$ to linked to ground. The process was initiated to generate a sine wave with the same periodicity and adequacy as the previous one. As can be found in Fig. 6 this yields the objective connected for the circuit for learning. In this manner, the system figured out how to synthesize the info sine wave as a yield. The system was built for a recurring sine wave with $10 \mathrm{MHz}$ frequency as well as 0.5 V.pp amplitude and is illustrated in Figure.10.The system consistently produced the desired frequency of $10 \mathrm{MHz}$. The output range is between $+.5 \mathrm{~V}$ to $-.5 \mathrm{~V}$. one percent adequacy error, the union time estimated was found to be $200 \mathrm{~ns}$ for $10 \mathrm{MHz}$. The Pulse single information given to the system was a heartbeat wave with $\mathrm{v} 1$ connected as .5 Vpp and recurrence $50 \mathrm{KHz}$ and $\mathrm{v}_{2}$ was associated with ground. The system was prepared for the sine wave yield with same recurrence and sufficiency. As can be found in the Fig. 6 the yield unmistakably pursues the objective connected for the circuit for learning. In a manner the system figured out how to recreate the info sine wave as a yield. The system was made to learn 10 $\mathrm{MHz}$ recurrence sine wave with. $5 \mathrm{~V}$ pp amplitude appeared in Fig.7. The system steadfastly imitated the ideal objective of $10 \mathrm{MHz}$ recurrence. The yield swing was from $+.5 \mathrm{~V}$ and $-.5 \mathrm{~V}$. The union time for $10 \mathrm{MHz}$ was determined as $200 \mathrm{~ns}$ with $1 \%$ blunder concerning sufficiency. The Neural system was tested for age of sine wave recurrence and sufficiency, more prominent than the info signal. Figure 24 demonstrates the outcome for the $100 \mathrm{KHz}$ yield created from a $50 \mathrm{KHz}$ information. The info $\mathrm{v}_{1}$ was a sine wave with $50 \mathrm{KHz}$ recurrence and .5 Vppsufficiency. The Neural Architecture is reached out for the use of picture pressure and decompression. The recreation result for picture pressure and decompression are appeared in the Fig. 14. The information $\mathrm{v}_{1}$ was a sine wave with $1 \mathrm{Vpp}$ voltage $5 \mathrm{MHz}$ recurrence and v2 was a sine wave with 
Table 1. Comparative table for the proposed work in terms of power, delay, and area with existing work

\begin{tabular}{|l|l|l|l|l|l|l|}
\hline \multirow{2}{*}{ Design } & \multicolumn{2}{l}{ Power } & \multicolumn{2}{l|}{ Delay (usec) } & \multicolumn{2}{l|}{ Area } \\
\cline { 2 - 8 } & Present & Existing & Present & Existing & $\begin{array}{l}\text { Present } \\
\text { (sqm) }\end{array}$ & $\begin{array}{l}\text { Existing } \\
\text { (sqm) }\end{array}$ \\
\hline Single Neuron [15] & $5.4 \mathrm{~mW}$ & $6.2 \mathrm{~mW}$ & 2.4 & 3.9 & 21.34 & 20.35 \\
\hline $\begin{array}{l}\text { Gilbert cell } \\
\text { multiplier [14,6] }\end{array}$ & $10.3 \mathrm{~mW}$ & $9.4 \mathrm{~mW}$ & 3.8 & 4.17 & 20.4 & 22.01 \\
\hline Compression [10] & $15 \mathrm{~mW}$ & $17 \mathrm{~mW}$ & 9.5 & 11.12 & 48.1 & 50.12 \\
\hline Decompression [12] & $9 \mathrm{~mW}$ & $12 \mathrm{~mW}$ & 13.1 & 14.5 & 47.23 & 47.35 \\
\hline Overall ANN [9,6] & $24 \mathrm{~mW}$ & $30 \mathrm{~mW}$ & 118 & 130 & 96.2 & 95.45 \\
\hline
\end{tabular}

\section{CONCLUSION}

Gilbert cell multiplier factor was laid out with info degree and farthest yield of $1.4 \mathrm{~V}$ Neuron cell. Activation result was intended for information shifts of $\pm 3 \mathrm{~V}$ as well as yield scope of 2.5 Uttermost separations existing yields fluctuate one small scale amperes. A Neural structure was propounded using these parts. The Neural structure chips away at the accessibility voltage $\pm 3 \mathrm{~V}$ with a yield swing of $\pm 2.8 \mathrm{~V}$. The Back Propagation (BP) programming algorithmic was utilized for training the network. For uncomplicated input with 1\% error, the proposed neural plan has a union time of 200ns. The Neural system was designed as a support for advanced and simple activities. The plan propounded will be utilized with the option existing engineering for the neural procedure. A feedforward coordinate that includes learning circuits as a well indistinct forward system that employs replica of loads for managing is included in the measured chip. The existence of systems with learning circuits has been acknowledged, and test results show that the learning circuits are effective without much of a stretch to become familiar with the info target fleeting sign. This design is effectively scaled to huge systems and is feasible for huge scale versatile sign handling. By implementing the circuits on MOSIS Tiny chips, the test results show that they are capable of performing their functions efficiently. Half pressure was accomplished utilizing propounded neural structure, a changed learning guideline is utilized to execute particular feed-forward ANNs with learning for use in versatile sign preparing.

\section{REFERENCES}

[1]. NOWAK, E. J. et al. Turning silicon on its edge (double gate CMOS/FinFET technology). IEEE Circuits and Devices Magazine, IEEE, Vol.20, PP. 20-31, 2004.

[2]. QU, J. et al. Study of drain induced barrier lowering (dibl) effect for strained si nmosfet. Procedia Engineering, Elsevier, Vol. 16, PP.298-305, 2011.

[3]. FOSSUM, J. G.; TRIVEDI, V. P. Fundamentals of Ultra-Thin-Body MOSFETs and FinFETs. [S.1.]: Cambridge University Press, 2013. ISBN 9781139343466.

[4]. ZHAO, W.; CAO, Y. Predictive technology model for nano-CMOS design exploration. ACM Journal on Emerging Technologies in Computing Systems (JETC), ACM, Vol.3, PP.1-9, 2007.

[5]. MARRANGHELlO, F. S.; REIS, A. I.; RIBAS, R. P. Design-oriented delay model for CMOS inverter. In: Integrated Circuits and Systems Design (SBCCI), 2012 25th Symposium on. [S.1.: s.n.], Vol.23, PP.1-6, 2012.

[6]. Sujata.A.et.al, "Performance Analysis Of Analog Data Compression And Decompression Using ANN In 32nm Finfet Technology", INTERNATIONAL JOURNAL OF SCIENTIFIC \& TECHNOLOGY RESEARCH VOLUME 8, ISSUE 12, DECEMBER 2019 ISSN 2277-8616. 
[7]. SAKURAI, T.; NEWTON, A. R. Alpha-power law MOSFET model and its applications to CMOS inverter delay and other formulas. IEEE Journal of solid-state circuits, IEEE, Vol. 25, PP.584-594, 1990.

[8]. NABAVI-LISHI, A.; RUMIN, N. C. Inverter models of cmos gates for supply current and delay evaluation. IEEE Transactions on Computer-Aided Design of Integrated Circuits and Systems, Vol. 13, PP. 12711279, Oct 1994.

[9]. DATTA, A. et al. Modeling and circuit synthesis for independently controlled double gate FinFET devices. IEEE transactions on computer-aided design of integrated circuits and systems, IEEE, v. 26, n. 11, p. 19571966, 2007.

[10]. DAGA, J. M.; AUVERGNE, D. A comprehensive delay macro modeling for submicrometer CMOS logics. IEEE Journal of Solid-State Circuits, IEEE, Vol. 34,PP. $42-$ $55,1999$.

[11]. ROSSELLÓ, J. L.; SEGURA, J. An analytical charge-based compact delay model for submicrometer cmos inverters. IEEE Transactions on Circuits and Systems I:Regular Papers, IEEE, Vol.51, PP. 13011311, 2004.

[12]. "http://www.itrs.net/," International Technology Roadmap for Semiconductors, 2013. 2, 44, 80, 81, 86

[13]. M. H. Abu-Rahma and M. Anis, Nanometer Variation-Tolerant SRAM Statistical Design for Yield. Vol.9, PP. 34-76, Springer, 2013.

[14]. S. Ghosh and K. Roy, "Parameter variation tolerance and error resiliency:New design paradigm for the nanoscale era," Proceedings of IEEE, Vol. 98, PP. 1718-1751, October 2010. 3, 17

[15]. S. Ganapathy, R. Canal, A. Gonzalez, and A. Rubio, "Circuit propagation delay estimation through multivariate regression-based modeling under spatio-temporal variability," in Design, Automation and Test in Europe Conference and Exhibition, 2010. 3, 28

[16]. S. Tawfik, Z. Liu, and V. Kursun, "Independent-gate and tied-gate finfet SRAM circuits:design guidelines for reduced area and enhanced stability," in Microelectronics, ICM Internatonal Conference on (ICM), 2007.

[17]. M. Fan, Y. Wu, V. Hu, P. Su, and C. Chuang, "Comparison of $4 \mathrm{t}$ and $6 \mathrm{t}$ finfet sram cells for subthreshold operation considering variability, a model-based approach," Electron Devices, IEEE Transactions on, Vol. 58, PP. 609-616, March, 03, 2011.

[18]. X. Wang, A. Brown, B. Cheng, and A. Asenov, "Statistical variability and reliability in nanoscale FinFET's," in Electron Devices Meeting (IEDM), IEEE International, 2011.

[19]. A. Asenov, A. Brown, J. Davies, S. Kaya, and G. Slavcheva, "Simulation of intrinsic parameter fluctuations in decananometer and nanometer-scale mosfets," IEEE transactions on electron devices, Vol. 50, PP. 1837-1852, September, 03, 2003.

[20]. E. Baravelli, M. Jurczak, N. Speciale, K. Meyer, and A. Dixit, "Impact of ler and random dopant fluctuations on finfet matching performance," IEEE transactions on nanotechnology, Vol. 7, PP. 291-298, March, 3, 2008.

[21]. N.Weste and D. Haris, CMOS VLSI Design. Pearson ddisonWesley, 2005

[22]. A. Agarwal, B. Paul, H. Mahmoodi, A. Datta, and K. Roy, "A processtolerant cache architecture for improved yield in nanoscale technologies," Very Large Scale Integration (VLSI) Systems, IEEE Transactions on, Vol. 13, PP. 27-38, January 2005.

[23]. M. Qazi, M. Sinangil, and A. Chandrakasan, "Challenges and directions for low-voltage sram," Design and Test of Computers, IEEE, Vol. 28, PP. 32-43, January 2018

[24]. K. Takeda, Y. Hagihara, Y. Aimoto, M. Nomura, Y. Nakazawa, T. Ishii, and $\mathrm{H}$. Kobatake, "A read-static-noise-margin-free sram cell for lowvdd and high-speed applications," Solid-State Circuits, IEEE Journal of, Vol. 41, PP. 113-121, January 2006.

[25]. B. Calhoun and A. Chandrakasan, "A 256kb $65 \mathrm{~nm}$ sub-threshold sram design for ultralow-voltage operation," Solid-State Circuits, IEEE Journal of, Vol. 42, PP. 680-688, March 2007.

\section{Creative Commons Attribution License 4.0 (Attribution 4.0 International, CC BY 4.0)}

This article is published under the terms of the Creative Commons Attribution License 4.0

https://creativecommons.org/licenses/by/4.0/deed.en_US 